\title{
Detection of Small-Scale Primary Users in Cognitive Radio Networks
}

\author{
Alexander W. Min, Student Member, IEEE, Xinyu Zhang, and Kang G. Shin, Fellow, IEEE
}

\begin{abstract}
In cognitive radio networks (CRNs), detecting smallscale primary devices, such as wireless microphones, is a challenging, but very important, problem that has not yet been addressed well. Recently, cooperative sensing and sensing scheduling have been advocated as an effective MAC (medium access control) layer approach to detecting large-scale primary signals. However, it is unclear whether and how they can improve the detection of a small-scale primary signal because of (i) its small signal footprint due to the use of weak transmit-power, and (ii) the unpredictability of its spatial and temporal spectrumusage patterns. Based on extensive analysis and simulation, we identify the data-fusion range as a key factor that enables effective cooperative sensing for detection of small-scale primary signals. In particular, we derive a closed-form expression for the optimal data-fusion range that minimizes the average detection delay. We also observe that the sensing performance is sensitive to the accuracy in estimating the primary's location and transmitpower. Based on these observations, we propose an efficient sensing framework that jointly performs Detection, LOCation estimation, and transmit-power estimation (DeLOC) for smallscale primary users. Our extensive evaluation results in a realistic CRN environment show that DeLOC achieves near-optimal detection performance, while meeting the detection requirements specified in the IEEE 802.22 standard draft. These findings provide useful insights and guidelines in designing a sensing scheme for detection of small-scale primaries in CRNs.
\end{abstract}

Index Terms-Cognitive radios, cooperative sensing, location and transmit-power estimation, small-scale primary users

\section{INTRODUCTION}

C OGNITIVE radio networks (CRNs) have recently been recognized as an attractive means to mitigate the spectrum scarcity problem that is expected to occur due to the rapidly growing wireless services and user population. In CRNs, unlicensed (secondary) devices can opportunistically access temporarily available licensed spectrum bands or white spaces, i.e., spectrum bands unoccupied by the primary users. As a first step towards realizing this new concept of opportunistic spectrum access, the FCC has recently approved the operation of unlicensed cognitive radio (CR) devices in UHF bands (a.k.a. TV white space) [1]. The first standardization effort based on this CR technology (i.e., the IEEE 802.22 wireless regional area networks (WRANs)) is also in its final stage [2]. Among the numerous challenges that CR technology

Manuscript received 1 December 2009; revised 25 May 2010 and 22 June 2010. A preliminary version of this work was presented at the 2010 IEEE INFOCOM Mini-conference. The work reported in this paper was supported in part by the NSF under Grant No. CNS-0721529.

The authors are with Department of Electrical Engineering and Computer Science, The University of Michigan, Ann Arbor, MI, 48109 USA (e-mail: \{alexmin,xyzhang,kgshin\}@eecs.umich.edu).

Digital Object Identifier 10.1109/JSAC.2011.110208. faces for its successful realization, spectrum sensing has been studied extensively as the key enabling technology.

Most of the previous research on spectrum sensing has focused on the various aspects of detecting large-scale primary signals (e.g., TV signals), including sensor cooperation [3], sensing scheduling [4], sensor mobility [5], and secure sensing [6], to name a few. The detection of a TV signal is relatively easy compared to that of a small-scale primary's signal. The predictable schedule, fixed location and high transmission power (e.g., $100 \mathrm{~kW}$ ) [7] of TV signals make a geo-location database a promising solution for TV signal detection [8]. Moreover, the digital TV (DTV) transition completed in June 2009 [9] leaves most of the UHF bands available to secondary users without the need for sensing TV signals.

On the other hand, detection of small-scale primary devices, such as wireless microphones (WMs), is very difficult and still remains to be an open problem for the following reasons. First, while a TV signal has a large transmission range (up to $150 \mathrm{~km}$ ), the WM signal has a small spatial footprint due to its weak transmission power (typically $10-50 \mathrm{~mW}$ ) [10]. This indicates that the 802.22 needs a separate dense sensor network for WM detection [7], or more preferably, an efficient cooperative sensing mechanism tailored to WM detection, which is the main focus of this paper. Second, the ON-OFF patterns of WMs have high spatial and temporal variations [11]. WMs can be turned on at any location and at any time without prior notification to secondary users. They are usually mobile and used at each location for a short period of time. Therefore, it is practically infeasible to maintain a database for WMs [8] or profile all the possible locations and schedules of WM usage in real time. More importantly, this unpredictability makes it hard for the base station (BS) to select proper sensors for cooperative sensing. Third, despite its small footprint, a WM must be detected according to the strict sensitivity requirement imposed by the FCC. For example, the 802.22 standard draft specifies that sensors must be able to detect as weak WM signals as $-114 \mathrm{dBm}$ over a $200 \mathrm{KHz}$ band within 2 seconds with both false-alarm and mis-detection probabilities less than 0.1. However, a recent measurement study [12] indicates that sensors suffer from a high falsealarm rate when detecting WM signals due to their weak signal strengths [13].

The detection of WMs is important for efficient spectrum utilization, especially in the space domain. For example, when a WM signal is detected by the sensors without knowing/estimating the transmitter's location, all the secondary users located within the cell of typical radius $33 \mathrm{~km}$ (up to $100 \mathrm{~km}$ ) may need to vacate the channel. Considering the small 
transmission range of a WM signal, i.e., $100-150 \mathrm{~m}$, this can cause significant under-utilization of spectrum in the space domain. Therefore, secondary users in 802.22 must be able to accurately detect the presence of a WM signal, and also estimate the WM transmitter's location.

Despite its practical importance, however, little has been done for the detection of small-scale primary signals. To the best of our knowledge, the disabling beacon protocol, recently proposed by the 802.22 Task Group 1 (TG 1) [14], [15], is the only known solution. The disabling beacon protocol aims to enhance WM detection by transmitting a specially-designed signal before starting the WM devices. It is suitable for carrying additional information, such as the signature/authentication and geo-location of WMs, which helps improve spectrum efficiency via better spatial [7], [16], [17] and frequency reuse [18]. However, the disabling beacon protocol still has the following limitations. First, we do not expect that all WM users will be equipped with a separate beacon device in the near future in view of the fact that most users have not even registered their WMs. Second, the transmit power of the beacon message is limited to the same level as the WM's (i.e., $250 \mathrm{~mW}$ in a UHF band), and thus, beacons cannot compensate for the low sensor density in 802.22 [14]. Finally, the disabling beacon protocol incurs a significant sensingtime overhead (i.e., 5-100 ms) [14] compared to simple energy detection, which may take only $1 \mathrm{~ms}$.

Motivated by these practical needs and problems, we propose an efficient sensing framework for detection of smallscale primaries using cooperative sensing. To cooperatively detect small-scale primary signals, the BS must carefully select a set of sensors by estimating the primary transmitter's characteristics, such as its location and transmit-power. We first assume this information is available to the secondary users, and derive the optimal fusion-range within which the sensors cooperate to minimize the detection delay, i.e., the number of sensing rounds needed for detecting a primary signal. Based on our analytical findings, we then designwithout assuming the availability of information on the primary transmitter's characteristics - a practical framework, called DeLOC, which performs joint cooperative sensing and location/transmit-power estimation, in order to meet the detectability requirements, while minimizing the detection delay.

This paper makes the following main contributions.

- Introduction of a novel spatio-temporal data-fusion scheme with the following salient features: it (i) exploits the physical-layer signal propagation characteristics in the space domain by finding an optimal fusion range for cooperative sensing, and (ii) makes statistics-based decisions in the time domain by identifying an optimal time to stop scheduling sensing. This spatio-temporal fusion provides useful and practical insights and can be used as a general framework for designing sensing schemes.

- Identification and characterization of the impacts of datafusion range and sensor density on the performance of small-scale primary detection in CRNs. We derive a closed-form expression for the optimal fusion range that minimizes the average detection delay. Moreover, we show that the optimal fusion range does not depend on sensor density and the minimum required sensor density for given detectability constraints decreases inversely proportional to the average detection delay.

- Development of a framework for joint small-scale primary detection and location/transmit-power estimation, called DeLOC. DeLOC iteratively performs cooperative sensing and location/transmit-power estimation until the fusion center (i.e., the BS) collects a sufficient amount of information to make a final decision. This approach allows the sensing and the estimation to refine each other over multiple scheduled periods.

- Design of a new data-fusion rule tailored to small-scale primary detection. Specifically, we propose a sequential probability ratio test with ascending weight (SPRT-AW) for DeLOC that intentionally delays the decision-making at the BS by assigning small weights to the decision statistics in early detection stages when location and transmit-power estimates are inaccurate. Our simulation results show that DeLOC combined with SPRT-AW achieves high detection accuracy, while minimizing the detection delay in a realistic 802.22 WRAN environment.

The remainder of this paper is organized as follows. Section II reviews the related work in detecting small-scale primary users/devices. Section III describes the network, signalpropagation and spectrum sensing models, and briefly introduces our approach to WM signal detection. Section IV studies the impact of data-fusion range on the performance of WM detection and location/transmit-power estimation. Section V formulates the sequential hypothesis testing problem for WM detection and derives an optimal fusion range that minimizes the average detection delay. Section VI details our proposed iterative sensing framework DeLOC which incorporates location and power estimation, and presents the SPRT-AW based datafusion rule. Section VII evaluates the performance of DeLOC, and Section VIII concludes the paper.

\section{RELATED WORK}

Despite its practical importance, there has only been limited research into MAC-layer solutions to WM detection. Most existing work focuses on PHY-layer signal detection techniques [19], [20], which have short sensing range and require a separate dense sensor network for WMs. Mishra et al. [7] studied the minimum sensor density required for detecting WM signals based on energy detection. They showed that when the path-loss exponent is 4 or higher, the average sensor density in rural areas (i.e., $1.25 / \mathrm{km}^{2}$ ) is not sufficient for detecting WMs. Recently, the 802.22 Working Group established Task Group 1 to develop a standard for the disabling beacon protocol [21]. Although the disabling beacon can protect WM signals better, it has several practical limitations as discussed in Section I. Moreover, the disabling beacon is restricted to WMs because the sensing relies on specialized signal features. In contrast, our DeLOC algorithm is a generic MAC-layer sensing scheme for small-scale primary detection, which can be incorporated into the beacon protocol while overcoming its limitations. Chouinard [17] proposed a coexistence model between WMs and 802.22 WRANs by exploiting the WM signal's small footprint and its narrow bandwidth, i.e., $200 \mathrm{KHz}$. However, 
they do not account for the location estimation, which greatly affects the spatial reuse of spectrum.

Sequential detection of primary users has been studied by others [22]-[24]. Chen et al. [24] proposed a weighted sequential probability ratio test (WSPRT) that assigns different weights to sensor reports based on the sensors' reputation in order to minimize the impact of the manipulated (or erroneous) sensor reports in making the final decision on the presence/absence of a primary signal. By contrast, SPRT-AW introduced in DeLOC is designed to intentionally defer the final decision at the BS, so as to reduce the effects of any wrong decision made in early stages when localization and power estimation are relatively inaccurate.

Chen et al. [25] proposed a scheme for verifying a primary user's location, called LocDef. Its main idea is that if the estimated location of the signal source differs significantly from the known location of the primary transmitter, i.e., a TV transmitter, then the BS assumes that the signal is transmitted from a fake primary user. By contrast, the location and transmit-power estimation introduced in DeLOC aim to improve the detection performance of small-scale primary signals, e.g., WMs, by helping the BS select an optimal set of cooperating sensors. In addition, when there is a WM signal, DeLOC returns the estimated location and transmitpower of the detected WM, so that the BS may use this information for admission control and transmit-power control of the secondary users to achieve better spectrum reuse in the space domain. Another key difference is that DeLOC makes use of a sparse sensor network, whereas LocDef requires a dense sensor network for location verification.

The work presented in this paper is also related to the existing body of research on the coverage of sensor networks. Using a theoretical analysis, Xing et al. [26] showed that data fusion via sensor cooperation can improve the coverage of sensor networks over the conventional detection schemes based on a disc model. While our approach also emphasizes the importance of data fusion, we adopt sensing scheduling to improve the detection performance of small-scale primaries. We characterize the impact of various factors on WM detection, and establish a practical framework that accounts for the unpredictability of each WM's transmit-power and location.

\section{Preliminaries}

In this section, we introduce the network model, the wireless signal-propagation model, the WM sensing model, and the data-fusion model.

\section{A. Network Model}

We consider a CRN consisting of primary and secondary users in the same geographical area. In general, there are two types of primary users: large-scale (e.g., TV transmitters) and small-scale (e.g., WMs). Here we focus on detecting smallscale primary users. While the techniques that we propose can be applied to other small-scale primary transmitters, we will focus on WM detection in IEEE 802.22 WRANs. WMs use a weak transmit power around $10-50 \mathrm{~mW}$, or below [10], [17], and its transmission range is only $150-200 \mathrm{~m}$, which is much smaller than the typical 802.22 cell radius of $33 \mathrm{~km}$.
We assume that WMs can use any UHF band and are turned on at random locations and at any time for relatively short periods of time. In 802.22, the secondary spectrum users are called consumer premise equipments (CPEs), which represent households in rural areas. Such CPEs are stationary and their locations are known to the BS. CPEs transmit/receive data to/from the BS, and function as spectrum sensors during the quiet periods reserved for primary detection. All the CPEs within the cell must be silent during quiet periods, and employ the spectrum sensors to measure the received signal strengths (RSSs) and report them to the BS for data-fusion. We assume secondary users have been deployed in an area $A$, i.e., an IEEE 802.22 WRAN cell, following a point Poisson process with density $\rho$, i.e., $n_{A} \sim \operatorname{Poi}(n ; \rho|A|)$. We assume a low sensor density $\rho$ as the typical density of CPEs (i.e., households) in rural areas is very low (around $1.25 / \mathrm{km}^{2}$ ) [27].

\section{B. Signal-Propagation and WM Sensing Models}

We assume that sensor $n$ 's received primary signal strength can be characterized by the following propagation model:

$$
P_{n}=P_{o}\left(\frac{d_{o}}{d_{n}}\right)^{\alpha} e^{X_{n}} e^{Y_{n}} \quad \text { (Watt), }
$$

where $d_{o}$ is the reference distance (e.g., $\left.1 \mathrm{~m}\right), P_{o}$ the received primary signal strength at the reference distance, $\alpha$ the path-loss exponent, and $d_{n}$ the distance from the primary transmitter to sensor $n$. Shadow fading and multi-path fading are accounted for in $e^{X_{n}}$ and $e^{Y_{n}}$, respectively, where $X_{n} \sim \mathcal{N}\left(0, \sigma^{2}\right) \forall n$. The log-normal shadow fading is often characterized by its $\mathrm{dB}$-spread, $\sigma_{d B}$, which has the relationship $\sigma=0.1 \ln (10) \sigma_{d B}$.

We make the following assumptions regarding the WM signal detection: Sensors

A1) use the energy detection for sensing, and

A2) sense an entire $6 \mathrm{MHz}$-wide TV channel.

Regarding A1, the feature detection cannot be applied for WM detection because, unlike the TV signals, there is no standard modulation specified by the FCC for WM signals [1]. The test statistic at sensor $n$ can be approximated as Gaussian using the Central Limit Theorem (CLT) as in [28]:

$$
T_{n} \sim \begin{cases}\mathcal{N}\left(N_{o}, \frac{N_{o}^{2}}{M_{s}}\right) & \mathcal{H}_{0} \text { (no primary signal) } \\ \mathcal{N}\left(P_{n}+N_{o}, \frac{\left(P_{n}+N_{o}\right)^{2}}{M_{s}}\right) & \mathcal{H}_{1} \text { (primary signal exists) }\end{cases}
$$

where $P_{n}$ is the power of a received primary signal at sensor $n, N_{o}$ the noise power, i.e., $-95.2 \mathrm{dBm}$ for a TV channel with $6 \mathrm{MHz}$ bandwidth [29], and $M_{s}$ the number of signal samples, e.g., $6 \times 10^{3} / \mathrm{ms}$ for $6 \mathrm{MHz} \mathrm{TV}$ band at the Nyquist rate.

Regarding A2, WMs use a relatively narrow frequency band, i.e., $200 \mathrm{KHz}$, compared to a $6 \mathrm{MHz} \mathrm{TV}$ band. Therefore, sensing the entire TV channel simplifies the sensing design at the cost of decreased measured signal-to-noise ratio (SNR) due to the increased noise level over a $6 \mathrm{MHz}$-wide channel.

At each sensing round (i.e., quiet periods), the BS directs a set of sensors to perform sensing for sensing duration of $T_{S}$ (e.g., $1 \mathrm{~ms}$ ), and the sensors report their readings to the BS for data fusion at the end of each sensing round. 


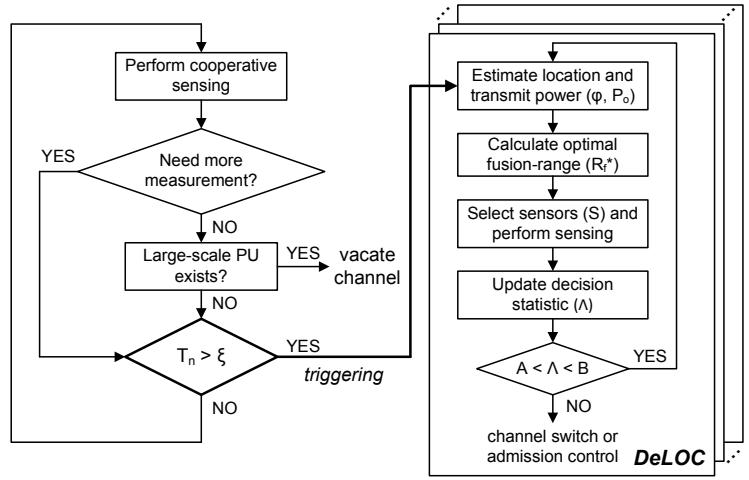

Fig. 1. The DeLOC framework: When a sensor reports a test statistic above a predefined threshold $(\xi)$, DeLOC for a small-scale primary, i.e., WM, is triggered by the BS that initiates and repeats the iteration between cooperative sensing and location/transmit-power estimation until the BS collects enough information for making a final decision.

\section{Data-Fusion Model}

For data fusion rule at the BS, we assume Equal Gain Combining (EGC) for a single-round sensing. EGC is known to have near-optimal performance without requiring the estimation of channel gains [30], and has the following decision statistic $T_{\Sigma} \triangleq \sum_{n=1}^{n_{s}} T_{n}$, where $T_{n}$ is the test statistic (i.e., measured RSS) of the energy detector at sensor $n$, and $n_{s}$ is the number of cooperative sensors. EGC will be used to characterize the impact of fusion range on detection performance (in Section IV). In DeLOC, the BS performs a sequential hypothesis testing for primary detection, and the test statistic of EGC, i.e., sum of the RSSs measured at cooperating sensors, will be used in updating the decision statistic for hypothesis testing (in Section V).

\section{The Proposed Approach}

Fig. 1 illustrates our proposed spectrum-sensing framework, DeLOC, which is tailored to the detection of small-scale primary signals such as WMs. When a large-scale primary signal exists, all the sensors within the network (e.g., an 802.22 cell) must vacate the channel regardless of the presence of small-scale primary signals. Thus, when a large-scale primary signal exists, DeLOC will not be triggered. ${ }^{1}$ To minimize the energy consumption and communication overhead, DeLOC for WM sensing is triggered only when a sensor reports a test statistic above a predefined threshold $(\xi)$ during the normal sensing mode for detection of large-scale primaries (i.e., TV signals). In our simulation study, we set $\xi=N_{o}+3.5 \sigma_{o}$ where $N_{o}$ and $\sigma_{o}=\frac{N_{o}}{\sqrt{M_{s}}}$ are the mean and standard deviation of the test statistics under $\mathcal{H}_{0}$. Note that the BS can run multiple instances of DeLOC in parallel corresponding to different triggering events at different geographical locations.

Upon triggering the detection process, the BS iteratively performs the location/transmit-power estimation and cooperative sensing until it collects a sufficient amount of information, i.e., measured test statistics, to make a final decision on the presence of a WM. In each sensing stage, the BS first estimates the location and transmit power of a WM, and based on this

\footnotetext{
${ }^{1}$ Note that large-scale primary signals can be reliably detected using either existing sensing schemes (e.g., [23]) or geo-location database [8]. The detection of large-scale primary signals is not within the scope of this paper.
}

estimation, it computes an optimal fusion range $\left(R_{f}^{*}\right)$ for cooperative sensing. Then, based on the test statistics reported from the sensors, the BS updates the decision statistic, and compares it with predefined lower $(A)$ and upper $(B)$ thresholds, to make a final decision. The thresholds are designed to guarantee the desired false-alarm and mis-detection probabilities (see Eq. (7) in Section V). If the test statistic is below the lower threshold, then the BS assumes the absence of a primary transmitter, e.g., the event was falsely triggered by the measurement error. If the test statistic exceeds the upper threshold, the BS assumes the presence of a primary transmitter at the estimated location, and then takes an appropriate action, e.g., vacating the channel or disabling nearby secondary users. Otherwise, the BS schedules another sensing event with the sensors within the optimal sensing range, thus accumulating the detection confidence in the temporal domain.

\section{Cooperative Sensing For SMAlL-SCALE Primary DETECTION}

In this section, we first study the impact of sensor cooperation on the detection of small-scale primary users. In particular, we investigate the impact of data-fusion range and localization error on the performance of signal detection.

\section{A. To Cooperate or Not?}

Although cooperative sensing is shown to help improve the sensing performance of large-scale primary users [5], [23], its relevance for small-scale primary detection is less obvious. On one hand, a large number of sensors may be needed for cooperation, because WM signals usually have a small footprint, and their spatial-temporal ON-OFF patterns are highly unpredictable. On the other hand, those sensors located far from the WM will report only the noise power. Thus, employing a large number of noisy reports may adversely affect the detection performance, since the energy detector cannot extract the primary signal from the noise. The set of sensors chosen for cooperative sensing may also affect the accuracy of location and transmit-power estimation, which play an important role in detecting WMs. In what follows, we thus investigate the impact of fusion range and location uncertainty on detection performance.

\section{B. Impact of Data-Fusion Range}

Fig. 2 shows the impact of data-fusion range on the detection probability $Q_{D}$ subject to a given false-alarm probability $Q_{F A}=0.01$ using MATLAB-based simulation. Intuitively, when the range is small, enlarging the range increases sensor diversity, thus improving the sensing performance. However, as the range increases further, the test statistics measured from the sensors become closer to the noise level, adversely affecting the detection performance. This implies the existence of an optimal fusion range that maximizes the sensing performance. Fig. 2 also indicates that the optimal range depends on the transmit power of the primary transmitter. (Also, see Fig. 5(a) in Section VII for more detail.) 


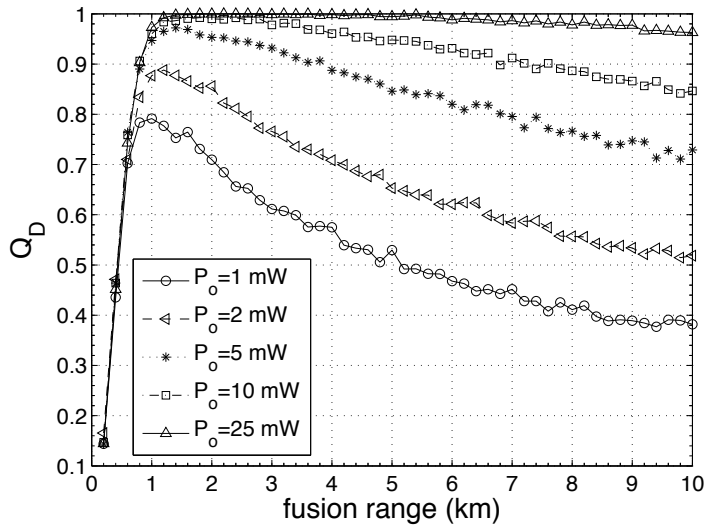

Fig. 2. Impact of data-fusion range: The figures show the existence of an optimal fusion range $R_{f}$ in terms of maximizing the detection probability $Q_{D}$. Sensor density, sensing duration, shadow fading dB-spread, path-loss exponent, and the required false-alarm probability are set to $\rho=1.25 \times$ $10^{-6} / \mathrm{m}^{2}, T_{S}=1 \mathrm{~ms}, \sigma_{d B}=5.5 \mathrm{~dB}, \alpha=4$, and $Q_{F A}=0.01$, respectively.

\section{Impact of Location-Estimation Error}

Ideally, the BS performs data fusion with a set of sensors located within the data-fusion range centered around the primary transmitter. In practice, however, the unpredictability of a primary's location can significantly degrade the quality of incumbent detection because it makes it difficult to select a proper set of sensors for data fusion.

Fig. 3 plots the WM detection probability $\left(Q_{D}\right)$ with onetime sensing for various location estimation errors. The figure shows that the detection performance degrades drastically as the localization error increases beyond a certain level, e.g., $1 \mathrm{~km}$. Even a small difference in one-time detection performance can greatly affect the average number of sensing rounds to achieve the desired false-alarm and mis-detection probabilities. Moreover, accurate location estimation is necessary for efficient co-existence between a WM and secondary users once DeLOC detects the presence of the WM signal. Therefore, reasonably accurate localization is necessary in our design of small-scale primary detection.

\section{Detection of Small-scale Primary via SPATIO-TEMPORAL DATA-FUSION}

In this section, we first formulate the small-scale primary detection problem as a sequential hypothesis testing problem. We then derive the optimal data-fusion range that minimizes the average detection delay. We finally show that the required sensor density to meet a certain detectability constraint decreases inversely proportional to the average number of sensing rounds scheduled for detection.

\section{A. Hypothesis Testing}

Let $\boldsymbol{\theta}_{t}=\left[T_{1}, \ldots, T_{\left|S_{t}\right|}\right]^{T}$ denote the vector of test statistics (i.e., RSSs) measured at the sensing stage $t$ by a set $S_{t}$ of cooperating sensors. A sensor is selected by the BS if it is within the fusion range $R_{f}$ from the WM transmitter. Note that the fusion range, and hence the set of cooperating sensors, can differ in each sensing stage according to the WM's estimated location and transmit-power level. Let $\boldsymbol{\theta}=\left[\boldsymbol{\theta}_{1}^{T}, \ldots, \boldsymbol{\theta}_{N}^{T}\right]^{T}$ denote the $M \times 1$ vector of test statistics measured at sensors

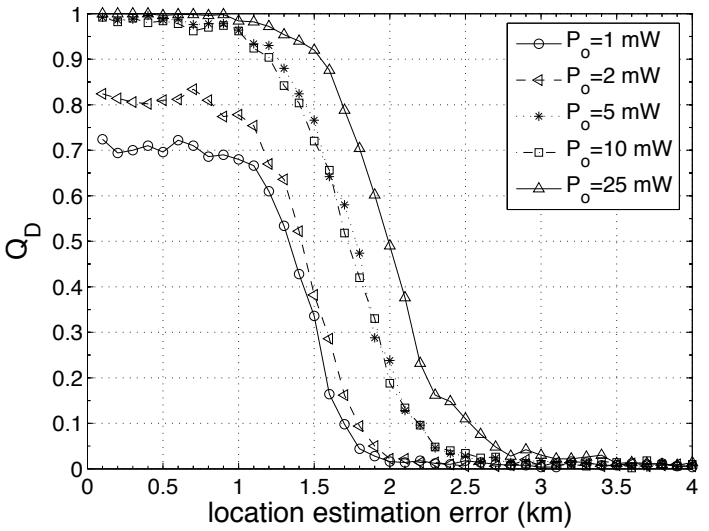

Fig. 3. Impact of localization error: The detection performance degrades as the location estimation becomes less accurate. The simulation parameters are set to $\rho=1.25 \times 10^{-6} / \mathrm{m}^{2}, T_{S}=1 \mathrm{~ms}, \sigma_{d B}=5.5 \mathrm{~dB}, \alpha=4, Q_{F A}=0.01$, and $R_{f}=2 \mathrm{~km}$, respectively.

over $N$ sensing stages, where $M=\sum_{t=1}^{N}\left|S_{t}\right|$. As shown in Eq. (2), the test statistics can be estimated to be Gaussian regardless of the existence of a primary signal [28].

Our detection problem is thus a binary Gaussian classification problem where the observed test statistic $\boldsymbol{\theta}$ belongs to one of two classes, $\mathcal{H}_{0}$ or $\mathcal{H}_{1}$, where:

$$
\begin{array}{ll}
\mathcal{H}_{0}: \boldsymbol{\theta} \sim \mathcal{N}\left(\boldsymbol{\mu}_{0}, \boldsymbol{\Sigma}_{0}\right) & \text { (no primary signal) } \\
\mathcal{H}_{1}: \boldsymbol{\theta} \sim \mathcal{N}\left(\boldsymbol{\mu}_{1}, \boldsymbol{\Sigma}_{1}\right) & \text { (primary signal exists), }
\end{array}
$$

where $\boldsymbol{\mu}_{k}$ and $\boldsymbol{\Sigma}_{k}$ are the mean vector and covariance matrix of the test statistics under $\mathcal{H}_{k}, k \in\{0,1\}$. The average test statistics under each hypothesis are $\boldsymbol{\mu}_{0}=N_{o} \times \mathbf{1}$ and $\boldsymbol{\mu}_{1}=$ $\left(P_{R}+N_{o}\right) \times \mathbf{1}$, where $N_{o}$ and $P_{R}$ are the average noise power and received primary signal power at sensors, respectively. ${ }^{2}$

The covariance matrix $\boldsymbol{\Sigma}_{0}$ can be expressed as $\boldsymbol{\Sigma}_{0}=\sigma_{o}^{2} \mathbf{I}$ where $\mathbf{I}$ is an $M \times M$ identity matrix and $\sigma_{o}^{2}=\frac{N_{o}^{2}}{M_{s}}$. Note that the correlation among sensor reports is negligible under the assumption that the locations of the sensors and WM transmitter are fixed during the detection process [23]. Moreover, in a very low SNR environment, it is reasonable to assume $P_{n}+N_{o} \approx N_{o} \forall n$, and hence the covariance matrix $\boldsymbol{\Sigma}_{1}$ can be approximated as $\boldsymbol{\Sigma}_{o} \approx \boldsymbol{\Sigma}_{1}=\sigma_{o}^{2} \mathbf{I}$.

\section{B. Sensing Scheduling via Sequential Probability Ratio Test}

In DeLOC, the BS schedules the sensing periods (stages) until it obtains a sufficient amount of information for making a final decision. Via sensing scheduling, the BS receives a sequence of test statistics $\left\{\boldsymbol{\theta}_{t}\right\}_{t=1}^{N}$ from the sensors. We adopt Wald's Sequential Probability Ratio Test (SPRT) [31] to process the statistics and determine when to stop sensing. SPRT is optimal in the sense of minimizing the average number of observations, given bounded false-alarm probability $Q_{F A}$ and mis-detection probability $Q_{M D}$. It enables the BS to reduce erroneous triggering of WM detection by optimizing its decision thresholds.

The decision statistic $\Lambda_{N}$ is the log-likelihood ratio derived from a sequence of test statistics $\boldsymbol{\theta}_{1}, \ldots, \boldsymbol{\theta}_{N}$ as follows:

\footnotetext{
${ }^{2}$ Since the BS does not have the exact distribution of the received primary signal strengths, the BS can set $P_{R}$ to $-107 \mathrm{dBm}$, which is the detectability requirement in 802.22 [14].
} 


$$
\Lambda_{N} \triangleq \lambda\left(\boldsymbol{\theta}_{1}, \ldots, \boldsymbol{\theta}_{N}\right)=\ln \frac{f_{1}\left(\boldsymbol{\theta}_{1}, \ldots, \boldsymbol{\theta}_{N}\right)}{f_{0}\left(\boldsymbol{\theta}_{1}, \ldots, \boldsymbol{\theta}_{N}\right)},
$$

where $f_{k}\left(\boldsymbol{\theta}_{1}, \ldots, \boldsymbol{\theta}_{N}\right)$ is the joint p.d.f. of the sequence of test statistics under the hypothesis $\mathcal{H}_{k} k \in\{0,1\}$.

With SPRT, a decision is made based on the observed sequence of test statistics, $\left\{\boldsymbol{\theta}_{t}\right\}_{t=1}^{N}$, using the following rules:

$$
\begin{aligned}
\Lambda_{N} \geq B & \Rightarrow \text { accept } \mathcal{H}_{1} \text { (primary signal exists) } \\
\Lambda_{N}<A & \Rightarrow \text { accept } \mathcal{H}_{0} \text { (no primary signal) } \\
A \leq \Lambda_{N}<B & \Rightarrow \text { take another observation, }
\end{aligned}
$$

where $A$ and $B(0<A<B<\infty)$ are the detection thresholds that depend on the desired values of $Q_{F A}$ and $Q_{M D}$.

Let $\lambda_{t}$ be the log-likelihood ratio at sensing stage $t$, i.e., $\lambda_{t}=\ln \frac{f_{1}\left(\boldsymbol{\theta}_{t}\right)}{f_{0}\left(\boldsymbol{\theta}_{t}\right)}$. Recall that $\left\{\boldsymbol{\theta}_{t}\right\}_{t=1}^{N}$ are Gaussian, and assume they are independent and identically distributed (i.i.d.). Then, Eq. (3) becomes:

$$
\Lambda_{N}=\sum_{t=1}^{N} \lambda_{t}=\sum_{t=1}^{N} \ln \frac{f_{1}\left(\boldsymbol{\theta}_{t}\right)}{f_{0}\left(\boldsymbol{\theta}_{t}\right)}=\sum_{t=1}^{N} \sum_{n=1}^{\left|S_{t}\right|} \ln \frac{f_{1}\left(\widetilde{T}_{n}\right)}{f_{0}\left(\widetilde{T}_{n}\right)}
$$

where the test statistic can be approximated as Gaussian using the Central Limit Theorem (CLT) as $\widetilde{T}_{n} \sim \mathcal{N}\left(\mu_{k}, \sigma_{o}^{2}\right)$ under $\mathcal{H}_{k}$, as shown in Eq. (2).

We now consider the normalized test statistics (i.e., RSSs) to simplify the derivation of the average number of sensing rounds. Let $\widetilde{T}_{n} \triangleq T_{n} \cdot \sigma_{o}^{-1}$ denote the normalized test statistic, i.e., $t_{n \mid \mathcal{H}_{k}} \sim \mathcal{N}\left(\phi_{k}, 1\right)$ where $\phi_{k}=\frac{\mu_{k}}{\sigma_{o}}, \forall k$. Then, we have:

$$
\lambda_{t}=\sum_{n=1}^{\left|S_{t}\right|} \ln \frac{h_{1}\left(\widetilde{T}_{n}\right)}{h_{0}\left(\widetilde{T}_{n}\right)}=\left(\phi_{1}-\phi_{0}\right) \sum_{n=1}^{\left|S_{t}\right|} \widetilde{T}_{n}+\frac{1}{2} \sum_{n=1}^{\left|S_{t}\right|}\left(\phi_{0}^{2}-\phi_{1}^{2}\right),
$$

where $h_{k}(\cdot)$ is the p.d.f. of $\widetilde{T}_{n \mid \mathcal{H}_{k}}$.

Based on Eqs. (4) and (5), the decision statistic $\Lambda_{N}$ can be expressed as:

$$
\begin{aligned}
\Lambda_{N} & =\left(\phi_{1}-\phi_{0}\right) \sum_{t=1}^{N} \sum_{n=1}^{\left|S_{t}\right|} \widetilde{T}_{n}+\frac{1}{2} \sum_{t=1}^{N} \sum_{n=1}^{\left|S_{t}\right|}\left(\phi_{0}^{2}-\phi_{1}^{2}\right) \\
& =\left(\phi_{1}-\phi_{0}\right) \sum_{n=1}^{M} \widetilde{T}_{n}+\frac{M}{2}\left(\phi_{0}^{2}-\phi_{1}^{2}\right),
\end{aligned}
$$

where $M=\sum_{t=1}^{N}\left|S_{t}\right|$ is the total number of test statistics collected by the BS through $N$ sensing stages.

SPRT can meet the desired detectability requirements by carefully setting the detection thresholds $A$ and $B$. Let $a^{*}$ and $b^{*}$ denote the desired values of $Q_{F A}$ and $Q_{M D}$, respectively. Then, the decision boundaries are given by [31]:

$$
A=\ln \frac{b^{*}}{1-a^{*}} \quad \text { and } \quad B=\ln \frac{1-b^{*}}{a^{*}},
$$

and the actual achievable error probabilities can only be slightly larger than the desired values $a^{*}$ and $b^{*}$.

\section{Minimization of the Average Detection Delay}

Recall that our goal is to minimize the number of sensing rounds that the $\mathrm{BS}$ has to schedule to meet the desired detection performance requirements, e.g., $Q_{F A}, Q_{M D} \leq 0.01$. Thus, we first derive a closed-form expression for the average number of sensing rounds required until a decision is made (i.e., either boundary $A$ or $B$ is reached).
The average number of sensing rounds required for making a decision (denoted by $\mathbb{E}[N]$ ) can be computed as [31]:

$$
\mathbb{E}[N]=\mathbb{E}\left[\lambda \mid \mathcal{H}_{k}\right]^{-1} \times \mathbb{E}\left[\Lambda_{N}\right] .
$$

First, using Eq. (5), the average value of the log-likelihood ratio $\lambda$ under hypothesis $\mathcal{H}_{k}$ can be derived as:

$$
\mathbb{E}\left[\lambda \mid \mathcal{H}_{k}\right]=\left(\phi_{1}-\phi_{0}\right) \mathbb{E}\left[\sum_{n=1}^{\left|S_{t}\right|} \widetilde{T}_{n \mid \mathcal{H}_{k}}\right]+\frac{1}{2} \mathbb{E}\left[\sum_{n=1}^{\left|S_{t}\right|}\left(\phi_{0}^{2}-\phi_{1}^{2}\right)\right] .
$$

Next, the expectation of $\Lambda_{N}$ in Eq. (8) can be found as follows. Suppose $\mathcal{H}_{1}$ holds, then $\Lambda_{N}$ will reach the decision boundary $A$ with the desired mis-detection probability $b^{*}$; otherwise, it will reach $B$. Thus, using Eq. (7), we have:

$$
\mathbb{E}\left[\Lambda_{N} \mid \mathcal{H}_{1}\right]=b^{*} \ln \frac{b^{*}}{1-a^{*}}+\left(1-b^{*}\right) \ln \frac{1-b^{*}}{a^{*}} .
$$

Based on Eqs. (8), (9) and (10), we can derive the average number of sensing rounds needed for decision-making as:

$$
\mathbb{E}\left[N \mid \mathcal{H}_{1}\right]=\frac{b^{*} \ln \frac{b^{*}}{1-a^{*}}+\left(1-b^{*}\right) \ln \frac{1-b^{*}}{a^{*}}}{\left(\phi_{1}-\phi_{0}\right) \mathbb{E}\left[\sum_{n=1}^{\left|S_{t}\right|} \widetilde{T}_{n \mid \mathcal{H}_{1}}\right]+\frac{1}{2}\left(\phi_{0}^{2}-\phi_{1}^{2}\right) \mathbb{E}\left[\left|S_{t}\right|\right]} .
$$

Similarly, the average number of sensing rounds under $\mathcal{H}_{0}$, i.e., $\mathbb{E}\left[N \mid \mathcal{H}_{0}\right]$, can be derived.

Eqs. (9), (10), and (11) indicate that the average number of sensing rounds $\mathbb{E}[N]$ depends on: (i) the average number of sensors within the fusion range, which can be easily calculated as $\mathbb{E}\left[\left|S_{t}\right|\right]=\rho \pi R_{f}^{2}$, under the assumption of the point Poisson distribution of sensors, i.e., $\left|S_{t}\right| \sim \operatorname{Poi}\left(n ; \rho \pi R_{f}^{2}\right)$, and (ii) the sum of their reported test statistics, i.e., $\mathbb{E}\left[\sum_{n=1}^{\left|S_{t}\right|} \widetilde{T}_{n \mid \mathcal{H}_{k}}\right]$.

As will be shown below, the sum of test statistics is affected mainly by three parameters: (i) sensor density $(\rho)$, (ii) transmit-power level of the primary device $\left(P_{o}\right)$, and (iii) data-fusion range $\left(R_{f}\right)$, assuming other parameters remain constant. In general, sensor density is known at the BS at the time of system deployment, and the transmit power can be estimated based on measurements (which will be detailed in Section VI-A). Therefore, we opt to derive an optimal fusion range $R_{f}^{*}$ that minimizes the average number of sensing rounds, thus minimizing the detection delay.

\section{Approximation of the Sum of Test Statistics}

Unfortunately, it is infeasible to derive a closed-form expression for the exact distribution of the sum of test statistics. This is because it depends on various random factors including the number of sensors within the fusion range, their locations relative to the primary transmitter, channel gains between the primary transmitter and the sensors, and the measurement error of the energy detector. Therefore, as a first step to derive an optimal fusion range, we approximate the sum of test statistics in Eq. (11) as a shifted log-normal random variable.

Let $T_{\Sigma\left(\rho, R_{f}\right)}$ denote the sum of the test statistics measured at the sensors within the fusion radius $R_{f}$ from the WM transmitter, in the network with sensor density $\rho$. Then, under $\mathcal{H}_{1}$, it can be approximated as: 


$$
\begin{aligned}
\mathbb{E}\left[T_{\Sigma\left(\rho, R_{f}\right)}\right] & =\mathbb{E}\left[\sum_{n \in S_{t}} T_{n \mid \mathcal{H}_{1}}\right] \\
& =\mathbb{E}\left[\sum_{n \in S_{t}} \mathcal{N}\left(P_{n}+N_{o}, \sigma_{o}^{2}\right)\right] \\
& \approx \mathbb{E}\left[\sum_{n \in S_{t}} P_{n}\right]+\mathbb{E}\left[\sum_{n \in S_{t}} N_{o}\right],
\end{aligned}
$$

where $P_{n}$ is the received primary signal strength at sensor $n$ and $S_{t} \equiv S\left(\rho, R_{f}\right)$ for brevity. The approximation in Eq. (12) is made based on the observation that, assuming the sensing duration of $1 \mathrm{~ms}$, the measurement errors of the energy detector is relatively smaller than the average received primary signal strength, i.e., $\sigma_{o}^{2} \ll\left(P_{n}+N_{o}\right)$.

Based on Eq. (12), we now focus on approximation of the sum of received primary signal strengths, which can be rewritten as $\mathbb{E}\left[\sum_{n \in S_{t}} P_{n}\right]=P_{o} \mathbb{E}\left[\sum_{n \in S_{t}} g\left(d_{n}\right) e^{X_{n}} e^{Y_{n}}\right]$ where $P_{o}$ is the primary's transmit power, $g\left(d_{n}\right)$ is the sensor $n$ 's channel gain due to path-loss, i.e., $g\left(d_{n}\right)=\left(d_{o} / d_{n}\right)^{\alpha}$, and $e^{X_{n}}$ and $e^{Y_{n}}$ are the channel gains from shadowing and multi-path fading, respectively. We approximate the sum of channel gains due to path-loss, denoted by $\mathcal{G}_{\Sigma}\left(\rho, R_{f}\right)=\sum_{n \in S_{t}} g\left(d_{n}\right)$, as a log-normal random variable. Previous numerical studies have shown that the aggregate interference of Poisson-distributed transmitters to a single receiver can be accurately approximated as a log-normal distribution [32]. Conversely, assuming the reciprocity of the RF path, we can also approximate the sum of received primary signal strengths at sensors as a lognormal random variable. It has been shown that the impacts of fading on received signal strengths is not a critical factor in such an approximation [33]. The effects of log-normal shadowing and multi-path fading in an average sense will be incorporated later (see Eq. (17)).

Denote $\mathcal{G}_{\Sigma}\left(\rho, R_{f}\right) \sim \log -\mathcal{N}\left(\mu_{G}, \sigma_{G}^{2}\right)$. Then, the p.d.f. of $\mathcal{G}_{\Sigma}\left(\rho, R_{f}\right)$ is given as:

$$
p_{\mathcal{G}\left(\rho, R_{f}\right)}(x)=\frac{1}{x \sigma_{G} \sqrt{2 \pi}} \exp \left(-\frac{\left(\ln x-\mu_{G}\right)^{2}}{2 \sigma_{G}^{2}}\right),
$$

where the $\mu_{G}$ and $\sigma_{G}^{2}$ have the following relationships [32]:

$$
m_{1}\left(\rho, R_{f}\right)=e^{\mu_{G}+\frac{1}{2} \sigma_{G}^{2}} \text { and } m_{2}\left(\rho, R_{f}\right)=e^{2 \mu_{G}+\sigma_{G}^{2}}\left(e^{\sigma_{G}^{2}}-1\right) .
$$

Here $m_{k}\left(\rho, R_{f}\right)$ is the $k$ th cumulant of $\mathcal{G}_{\left(\rho, R_{f}\right)}$, given as:

$$
\begin{aligned}
m_{k}\left(\rho, R_{f}\right) & =\rho \pi\left(R_{f}^{2}-\epsilon^{2}\right) \int_{\epsilon}^{R_{f}} \frac{2 r}{\left(R_{f}^{2}-\epsilon^{2}\right)} g(r)^{k} \mathrm{~d} r \\
& =\frac{2 \rho \pi d_{o}^{k \alpha}}{(k \alpha-2)}\left(\frac{1}{\epsilon^{k \alpha-2}}-\frac{1}{R_{f}^{k \alpha-2}}\right)
\end{aligned}
$$

where $d_{o}$ is the reference distance and $\epsilon$ is the minimum separation between the primary transmitter and the sensors, which is set to $\epsilon=75 \mathrm{~m}$ in our simulation. ${ }^{3}$

From Eqs. (14) and (15), the log-normal random variable $\mathcal{G}_{\Sigma}\left(\rho, R_{f}\right) \sim \log -\mathcal{N}\left(\mu_{G}, \sigma_{G}^{2}\right)$ can be approximated as:

$$
\mu_{G}=\frac{1}{2} \ln \left(\frac{m_{1}^{4}}{m_{1}^{2}+m_{2}}\right) \quad \text { and } \quad \sigma_{G}^{2}=\ln \left(1+\frac{m_{2}}{m_{1}^{2}}\right) .
$$

\footnotetext{
${ }^{3}$ This is reasonable because the probability that there exists at least one sensor within $\epsilon=75 \mathrm{~m}$ from the WM transmitter is $1-\operatorname{Poi}\left(0 ; \rho \pi \epsilon^{2}\right) \approx 0.02$ given sensor density of $\rho=1.25 \times 10^{-6} / \mathrm{m}^{2}$.
}

Therefore, from Eqs. (12) and (16), and by incorporating the effects of shadowing and multi-path fading assuming the fading is i.i.d. for each sensor, the sum of received primary power at the cooperating sensors $S_{t}$ can be expressed as:

$$
\mathbb{E}\left[\sum_{n \in S_{t}} P_{n}\right]=P_{o} \cdot \mathbb{E}\left[e^{X}\right] \cdot \mathbb{E}\left[e^{Y}\right] \cdot \mathbb{E}\left[\mathcal{G}_{\Sigma}\left(\rho, R_{f}\right)\right],
$$

where $\mathbb{E}\left[e^{X}\right]=e^{\frac{1}{2} \sigma^{2}}, \sigma=0.1 \ln (10) \sigma_{d B}$, and $\mathbb{E}\left[\mathcal{G}_{\Sigma}\left(\rho, R_{f}\right)\right]=$ $e^{\mu_{G}+\frac{1}{2} \sigma_{G}^{2}}$. For multi-path fading, we assume Rayleigh fading with zero mean, and thus, $\mathbb{E}\left[e^{Y}\right]=1$.

Then, from Eqs. (12) and (17), the average of the sum of normalized test statistics can be expressed as:

$$
\begin{aligned}
\mathbb{E}\left[\sum_{n=1}^{\left|S_{t}\right|} \widetilde{T}_{n \mid \mathcal{H}_{1}}\right] & =\mathbb{E}\left[T_{\Sigma\left(\rho, R_{f}\right)} \sigma_{o}^{-1}\right] \\
& =\left(P_{o} e^{\frac{1}{2} \sigma^{2}} \mathbb{E}\left[\mathcal{G}_{\Sigma}\left(\rho, R_{f}\right)\right]+N_{o} \rho \pi R_{f}^{2}\right) \sigma_{o}^{-1} .
\end{aligned}
$$

Finally, based on Eqs. (9) and (18), the first term in Eq. (8) for calculating the average number of sensing rounds $\mathbb{E}\left[N \mid \mathcal{H}_{1}\right]$ can be derived as:

$$
\begin{aligned}
\mathbb{E}\left[\lambda \mid \mathcal{H}_{1}\right] & =\frac{1}{2}\left(\phi_{0}^{2}-\phi_{1}^{2}\right) \rho \pi R_{f}^{2}+\left(\phi_{1}-\phi_{0}\right) \\
& \times\left(P_{o} e^{\frac{1}{2} \sigma^{2}} \mathbb{E}\left[\mathcal{G}_{\Sigma}\left(\rho, R_{f}\right)\right]+N_{o} \rho \pi R_{f}^{2}\right) \sigma_{o}^{-1}
\end{aligned}
$$

where $\phi_{0}=\frac{N_{o}}{\sigma_{o}}$ and $\phi_{1}=\frac{N_{o}+P_{R}}{\sigma_{o}}$ are the average normalized test statistics under both hypotheses.

The average number of sensing rounds $\mathbb{E}\left[N \mid \mathcal{H}_{1}\right]$ can be derived by substituting Eqs. (10) and (19) into Eq. (8).

\section{E. Optimal Data-Fusion Range}

Based on the analyses above, we now derive an optimal data-fusion range that minimizes the average detection delay, i.e., the number of sensing rounds needed to meet the detection performance requirements.

Proposition 1 Let $\mathcal{J}\left(R_{f}\right) \triangleq \mathbb{E}\left[\lambda \mid \mathcal{H}_{1}\right]$ in Eq. (19). Then, the optimal fusion range that minimizes the average number of sensing rounds $\mathbb{E}[N]$ is given as:

$$
R_{f}^{*}=\arg \max _{R_{f}} \mathcal{J}\left(R_{f}\right)=\left.R_{f}\right|_{\frac{\partial J\left(R_{f}\right)}{\partial R_{f}}=0}=\left(\frac{a_{1}(\alpha-2)}{2 a_{2}}\right)^{\frac{1}{\alpha}},
$$

where

$$
\begin{aligned}
a_{1} & =\frac{2\left(\phi_{1}-\phi_{0}\right) P_{o} e^{\frac{1}{2} \sigma^{2}} \rho \pi d_{o}^{\alpha}}{\sigma_{o}(2-\alpha)}, \\
a_{2} & =\frac{1}{2}\left(\phi_{0}^{2}-\phi_{1}^{2}\right) \rho \pi+\frac{\left(\phi_{1}-\phi_{0}\right) N_{o} \rho \pi}{\sigma_{o}} .
\end{aligned}
$$

Proof. See Appendix A.

Proposition 1 indicates that the optimal fusion range that minimizes the detection delay depends on various system parameters, such as transmission power $\left(P_{o}\right)$, noise power $\left(N_{o}\right)$, shadow fading $(\sigma)$, and path-loss exponent $(\alpha)$.

Based on Proposition 1, we have the following counterintuitive observation:

Corollary 1 The optimal fusion range $\left(R_{f}^{*}\right)$ is independent of the sensor density $\rho$.

One might think that the optimal fusion range should decrease as the sensor density increases, since more sensors (near 
the WM transmitter) with high RSSs become available for data fusion. However, this is not the case because the performance of EGC depends on how far a cooperating sensor's report (i.e., the measured RSS) is from the noise power level, which is independent of the sensor density (see Fig. 6 for details).

\section{F. Impact of Sensing Scheduling}

We now show that sensing scheduling can reduce the minimum sensor density required for given detection constraints. While the achievable performance gain via cooperative sensing has been studied extensively [5], [23], [34], the impact of sensing scheduling on sensor density requirement has not been studied before.

Proposition 2 The minimum sensor density required to meet certain detectability requirements is inversely proportional to the average number of times to sense.

Proof. Based on Eqs. (8), (10), (15) and (19), the required sensor density for a given average number of sensing rounds $\mathbb{E}[N]$ can be expressed as:

$$
\rho=\frac{\sigma_{o}^{2}}{\mathbb{E}[N]} \times \frac{b^{*} \ln \frac{b^{*}}{1-a^{*}}+\left(1-b^{*}\right) \ln \frac{1-b^{*}}{a^{*}}}{P_{R}\left[P_{o} e^{\frac{1}{2} \sigma^{2}}\left(\frac{1}{\epsilon^{2}}-\frac{1}{R_{f}^{2}}\right)-\frac{1}{2} \pi R_{f}^{2} P_{R}\right]},
$$

where $a^{*}$ and $b^{*}$ are the desired false-alarm and mis-detection probability values. Eq. (21) indicates that the sensor density $\rho$ is inversely proportional to the average number of sensing rounds. Therefore, the proposition follows.

Proposition 1 and 2 are derived based on the assumption that the WM's location and transmit-power level are known a priori to the secondary users. However, such information may not available in practice, and thus the benefits of our analytical findings cannot be realized without an efficient way of estimating the WM's location and transmit power. This motivates our approach of integrating sensing with location and transmit-power estimation, which we discuss next.

\section{DeLOC: THE ITERATIVE APPROACH}

We now introduce DeLOC, an iterative algorithm that expedites the detection of small-scale primary signals via joint data-fusion and location/transmit-power estimation. We first describe the estimation techniques, and then the proposed datafusion rule and the iteration method employed by DeLOC.

\section{A. Estimation Techniques}

1) Estimation of WM Location: As we observed in Fig. 3, a reasonable approximation, if not accurate, of the WM's location is sufficient for improving the detection performance. In DeLOC, the BS estimates and updates the WM's location based on the RSSs reported by the sensors. In particular, the BS employs a weighted centroid method proposed in [35], which estimates the WM's location via a weighted average of the sensors' locations, where the weight equals the corresponding sensor's report. The BS further refines the estimation via an exponential moving average over multiple sensing stages.

More specifically, let $\widehat{\vartheta}_{t}=\left(\widehat{x}_{t}, \widehat{y}_{t}\right) \in \mathbb{R}^{2}$ denote the estimated location of the primary at sensing stage $t$. Then, the WM's location is estimated as [35], [36]:

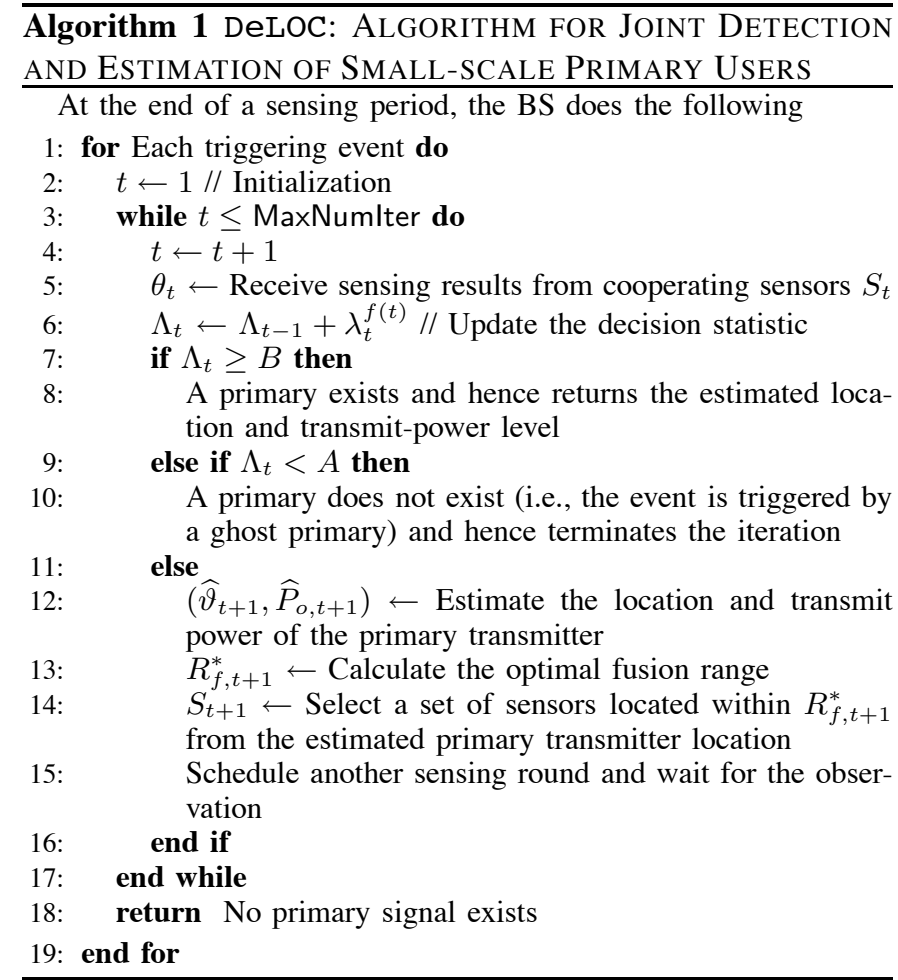

$$
\widehat{\vartheta}_{t}=(1-\beta) \widehat{\vartheta}_{t-1}+\beta\left(\sum_{n \in S_{t}} \frac{P_{n}}{\sum_{m \in S_{t}} P_{m}} \psi_{n}\right),
$$

where $P_{n}$ is the received primary signal power at sensor $n$, $\psi_{n}=\left(x_{n}, y_{n}\right)$ the location of sensor $n$, and $\beta \in(0,1)$ the smoothing factor.

Note that DeLOC uses a simple existing localization method to estimate the PU's location in each round, but it is not restricted to any specific localization algorithm, so other localization methods such as the semi range-based method proposed by Ma et al. [37], can also be used.

2) Estimation of Transmit Power: In DeLOC, the BS estimates the WM's transmit-power based on the WM's estimated location and the reported RSSs using the method proposed in [38] as:

$\widehat{P}_{o, t}(d B)=10 \log _{10}\left(\frac{1}{k}\right)+\frac{10}{\left|S_{t}\right|} \sum_{n \in S_{t}}\left(\log _{10}\left(P_{n}\right)+\alpha \log _{10}\left(d_{n}\right)\right)$, where $k=p_{o} d_{o}^{\alpha} P_{o}^{-1} \cdot p_{o}$ is the measured signal power at reference distance $d_{o}, P_{n}$ the received primary signal strength at sensor $n$, and $d_{n}$ the distance between the WM transmitter and sensor $n$, i.e., $d_{n}=\sqrt{\left(\hat{x}_{t}-x_{n}\right)^{2}+\left(\hat{y}_{t}-y_{n}\right)^{2}}$. Note that the test statistics of the energy detector include noise power, so the received primary signal strength $P_{n}$ needs to be estimated from the test statistics by subtracting the average noise power $N_{o}$ from the measurements.

While DeLOC employs simple location and power estimation techniques, the estimation accuracy can be further improved by using more sophisticated techniques at the cost of more computation.

\section{B. The Proposed Data-Fusion Rule}

While DeLOC improves the small-scale primary detection performance via iterative cooperative sensing and estimation, 
we observed that it often terminates in early stages misdetecting the WM. This is because, initially, the BS's location and transmit-power estimates are inaccurate, resulting in many noisy sensor reports during data fusion. This prevents DeLOC from fully exploiting its unique feature - an iterative refinement of the estimation and fusion.

To overcome this problem, we propose a new data-fusion rule for DeLOC, a sequential probability ratio test with ascending weight (SPRT-AW), to prevent the BS from making biased decisions in early stages. The idea is to assign smaller weights to the decision statistics in early stages, and gradually increase the weights as the location and transmitpower estimates become more accurate. Specifically, we use the following rule to update the decision statistic:

$$
\Lambda_{t}=\Lambda_{t-1}+\lambda_{t}^{f(t)} \quad \text { where } \quad f(t)=\frac{1}{1+e^{1-t}} \quad t \in \mathbb{N},
$$

where we use the sigmoid function $f(t)$ such that the exponent of test statistics increases from 0.5 to 1 as $t$ increases. Consequently, the test statistics in later stages count more in decision-making. The resulting decision statistics will be used in updating the $\Lambda_{t}$ in Eq. (4), and compared with the upper $(B)$ and lower $(A)$ thresholds to make a decision.

\section{Description of DeLOC Protocol}

As described in Fig. 1, DeLOC is triggered only when a sensor's report is above a certain predefined threshold $\xi$, which is suspected as a WM signal. The triggering threshold must be chosen carefully by the BS to balance the false- and mistriggering of DeLOC. Upon triggering, the BS assumes the triggering sensor's location as the WM's location, and employs additional sensors within the fusion range for WM detection in the next scheduled sensing round. If there are multiple triggering sensors in close proximity, the BS considers the sensor with highest RSS. In each sensing round, the BS iterates the following two steps: (i) location and transmit-power estimation and (ii) data-fusion, until the decision statistic for data fusion $\Lambda$ reaches one of the thresholds. The BS also terminates the iteration after scheduling sensing rounds for MaxNumlter. Algorithm 1 details DeLOC.

\section{PERformance Evaluation}

In this section, we evaluate DeLOC using MATLAB-based simulation. We first describe the simulation setup and then present the impact of fusion range on detection performance and its dependency on the transmit-power. We also show the relationship between the detection delay and sensor density. Finally, we demonstrate the performance of DeLOC in comparison with other testing schemes.

\section{A. Simulation Setup}

In the simulation, we consider a realistic 802.22 environment where sensors are randomly distributed over a $30 \mathrm{~km} \times 30 \mathrm{~km}$ area. The average sensor density is set to $1.25 / \mathrm{km}^{2}$, as typically used in 802.22 WRANs [7], unless specified otherwise. We assume a WM randomly located in the area with effective transmit-power below $25 \mathrm{~mW}$, as indicated by the measurement study in [14]. The maximum number of sensing rounds scheduled within the 2-second channel detection period (CDT) is limited to MaxNumlter $=100 .^{4}$ The duration of a single sensing period is assumed to be $1 \mathrm{~ms}$. The path-loss exponent is $\alpha=4$, and the shadow fading dB-spread is $\sigma_{d B}=5.5 \mathrm{~dB}$, which is typically assumed for rural areas. We also assume the signal-propagation parameters are known a priori to the secondary system. The triggering threshold in DeLOC is configured as $\xi=N_{o}+3.5 \sigma_{o}$, which gives the false-triggering rate of $2.3 \times 10^{-4}$. The simulation results are obtained from $5 \times 10^{3}$ randomly-generated topologies.

To evaluate the efficacy of DeLOC, we compare the performance of the following four sensing schemes: (i) Oracle (the ideal case), (ii) DeLOC with SPRT-AW, (iii) DeLOC, (iv) DeLOC without localization, and (v) DeLOC without transmitpower estimation. In Oracle, the location and transmit-power information is available to the BS, so the BS always uses the optimal fusion range for sensing without the need for estimation. Thus, Oracle will be used as a performance reference. In DeLOC without localization, the location of the triggering sensor is regarded as the primary's location. In DeLOC without power estimation, the power level is assumed to be randomly chosen in $[0,25] \mathrm{mW}$.

\section{B. Impact of Fusion Range}

Fig. 4 shows the impact of data fusion range on the detection performance in terms of detection delay and accuracy. The figures indicate that too small a fusion range suffers from the lack of cooperating sensors, which makes it difficult for the BS to collect enough information, i.e., measured RSSs, to make a decision within MaxNumlter, resulting in a low detection probability. On the other hand, too large a fusion range, i.e., beyond $1 \mathrm{~km}$, suffers from having many noisy reports, misleading the $\mathrm{BS}$ to promptly conclude that there is no primary signal, increasing the chance of misdetection. Fig. 4(b) shows that the detection probability $Q_{D}$ is maximized when the fusion range is $1 \mathrm{~km}$, which closely match the analytical result, i.e., $1.03 \mathrm{~km}$.

An additional observation from our simulation results is that false-alarms occur only 16 times over $5 \times 10^{3}$ iterations, i.e., $Q_{F A}=16 / 5000=0.0032$, thus achieving the false-alarm requirement of $Q_{F A} \leq 0.01$.

\section{Optimal Fusion Range}

Fig. 5(a) plots the optimal fusion range for various transmitpower levels, and indicates that our analytical results (Proposition 1 in Section V-E) closely match the simulation results. The figure also shows that to detect a high transmit-power $\mathrm{WM}$, it is better (in the sense of reducing the sensing delay) to extend the fusion range, thus increasing the number of cooperating sensors. On the other hand, to detect a WM with very weak transmit-power, it is better to have a small number of sensors, thus reducing the number of noisy reports. Fig. 5(b) indicates that the optimal fusion range (in the sense of maximizing the detection probability $Q_{D}$ ) remains the same over different sensor densities, thus confirming Corollary 1 in Section V-E.

\footnotetext{
${ }^{4}$ This is reasonable since the BS can schedule sensing as frequently as once every $10 \mathrm{~ms}$, i.e., one MAC frame size in 802.22.
} 


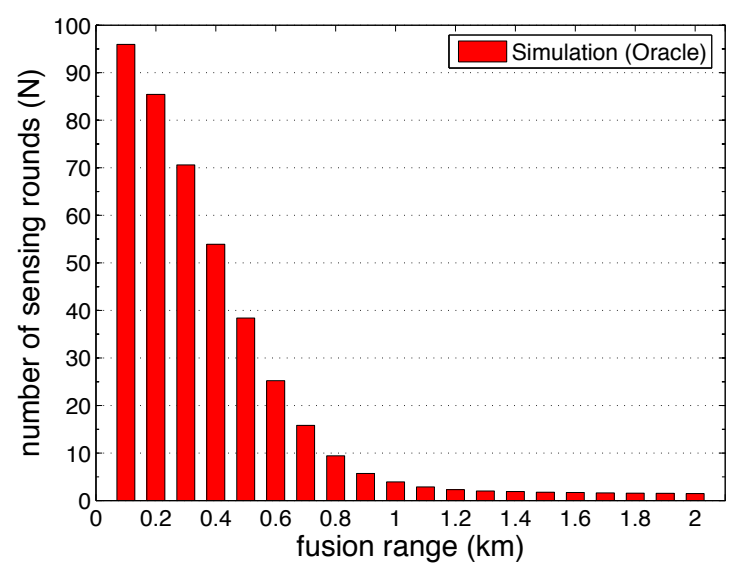

(a) average number of sensing rounds $(N)$

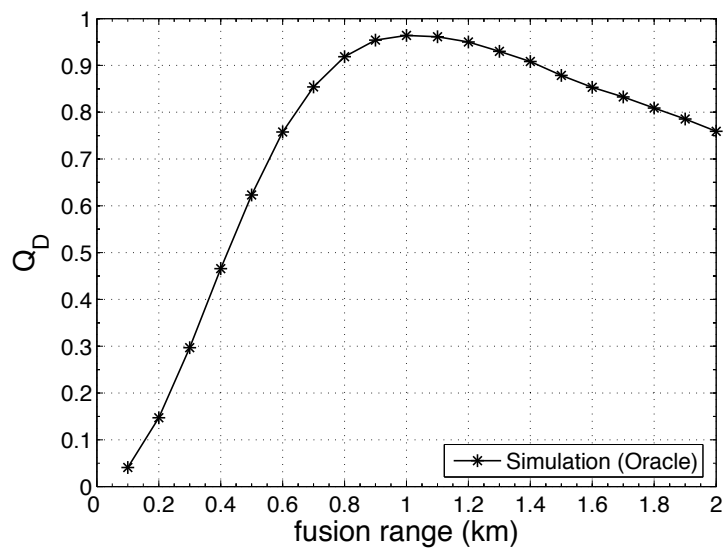

(b) detection probability $\left(Q_{D}\right)$

Fig. 4. Impact of data-fusion range on detection performance: (a) the average sensing rounds decreases as the fusion range increases, whereas (b) the detection probability is maximized when $R_{f}=1 \mathrm{~km}$, which is close to the analytical result. Here the WM's transmit power is set to $1 \mathrm{~mW}$.

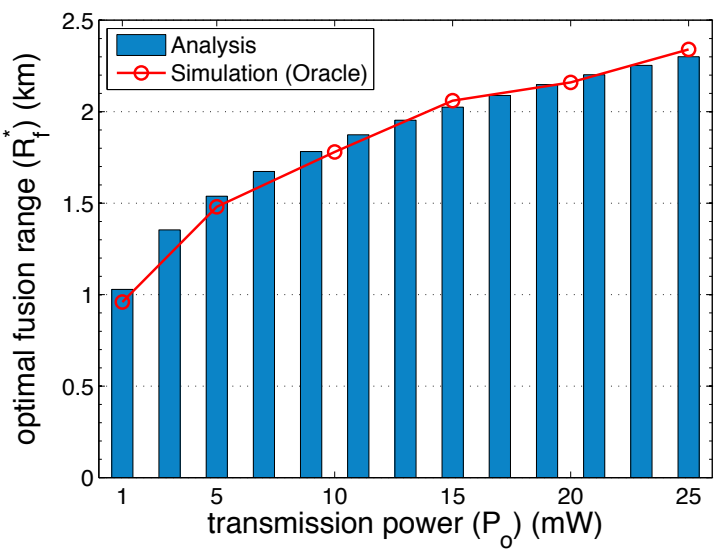

(a) Impact of transmit-power

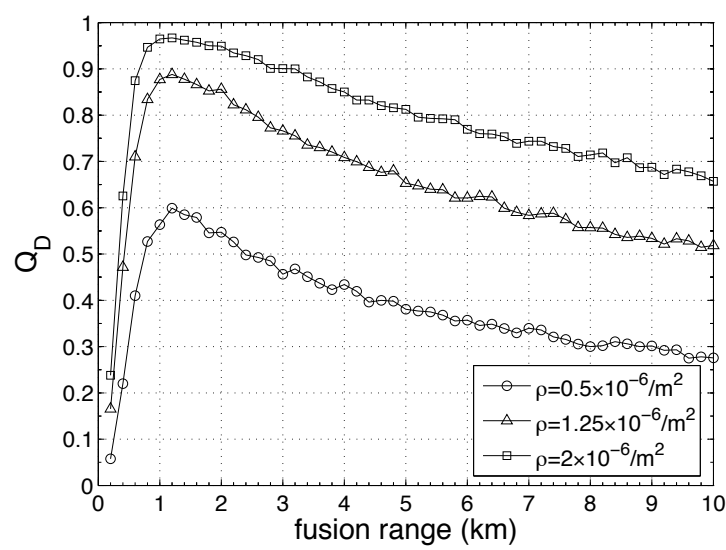

(b) Impact of sensor density

Fig. 5. Optimal Fusion Range: (a) shows the impact of transmit-power on the optimal fusion range, indicating that the analytical results (the bar graph) closely match the simulation results (the line graph), thus validating the analytical results. (b) shows that the optimal fusion range $R_{f}^{*}$ that maximizes the detection performance $\left(Q_{D}\right)$ does not depend on sensor density.

\section{Impact of Sensor Density}

Although sensor density does not affect the optimal fusion range, a higher sensor density (hence more sensors within fusion range) can still improve the sensing performance by exploiting diversity of measurement. Fig. 6(a) shows the average number of sensing rounds (i.e., detection delay) required to meet the detection performance $Q_{F A}, Q_{M D} \leq 0.01$, which obviously decreases with sensor density. The figure also indicates that the average number of sensing rounds is almost inversely proportional to the sensor density, thus confirming Proposition 2 in Section V. Fig. 6(b) further shows that the detection probability $Q_{D}$ increases with increasing sensor density.

\section{E. Performance of DeLOC}

To demonstrate the efficacy of DeLOC, we compare its performance with the other four testing schemes under the detection constraints $Q_{F A}, Q_{M D} \leq 0.01$. As shown in Fig. 7, when the WM's transmit-power increases, the detection performance (with respect to delay and detection probability) increases for all testing schemes. We make three additional observations.
First, Fig. 7(a) shows that the average number of sensing rounds for decision-making is below 10, which may take only $100 \mathrm{~ms}$ as the BS can schedule sensing periods as frequently as every $10 \mathrm{~ms}$, i.e., one MAC frame size in 802.22. In addition, the detection probability of DeLOC with SPRT-AW meets the detection requirement of 802.22 , i.e., $Q_{M D} \leq 0.1$, even for a very weak transmit-power of $1 \mathrm{~mW}$, as indicated in Fig. 7(b).

Second, Fig. 7(b) shows that DeLOC with SPRT-AW performs close to Oracle in terms of detection rate, and outperforms all other schemes that use regular SPRT. As mentioned earlier, the SPRT in DeLOC often makes a wrong decision (mis-detection of a WM) in early detection stages based on many noisy reports due to the inaccurate location and power estimates. DeLOC with SPRT-AW mitigates this problem by discounting the decision statistics in early stages.

Third, Fig. 7(b) shows that DeLOC without localization outperforms the one without transmit-power estimation. This is because the power estimation plays an important role in finding the optimal fusion range, and thus, the error in power estimation results in significant performance degradation. On the other hand, the location-estimation error is small compared with the typical fusion range, and thus it does not cause significant performance degradation, as already shown in Fig. 3. 


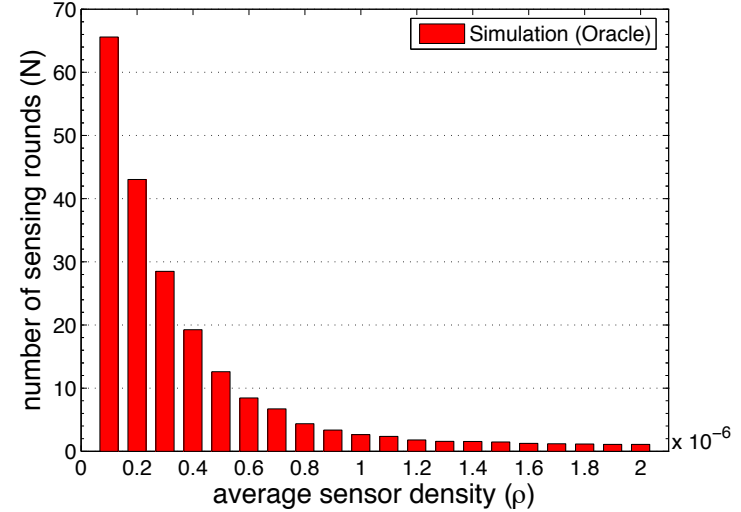

(a) Impact of sensor density on detection delay

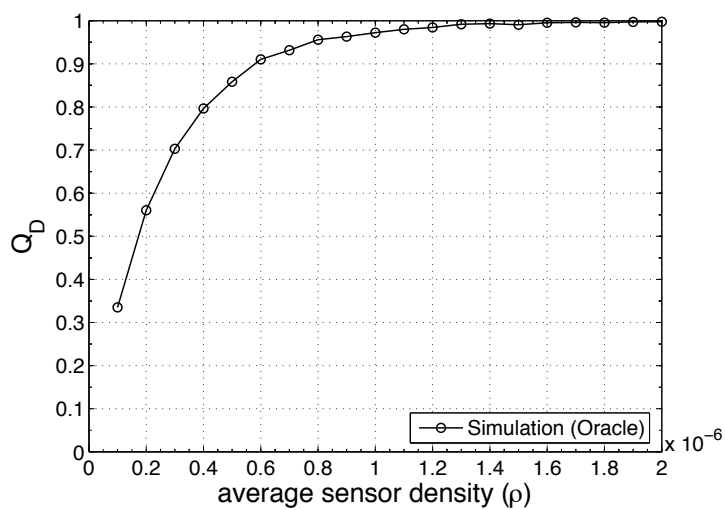

(b) Impact of sensor density on detection accuracy

Fig. 6. Impact of sensor density on detection performance: (a) the detection delay decreases rapidly as the sensor density increases, and (b) the detection accuracy increases as the sensor density increases. In the simulation, the transmit power is set as $P_{o}=2 \mathrm{~mW}$.

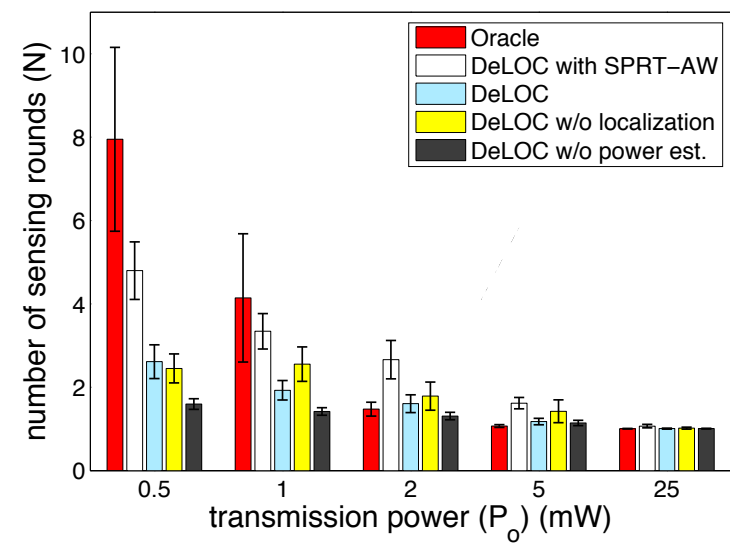

(a) Average detection delay

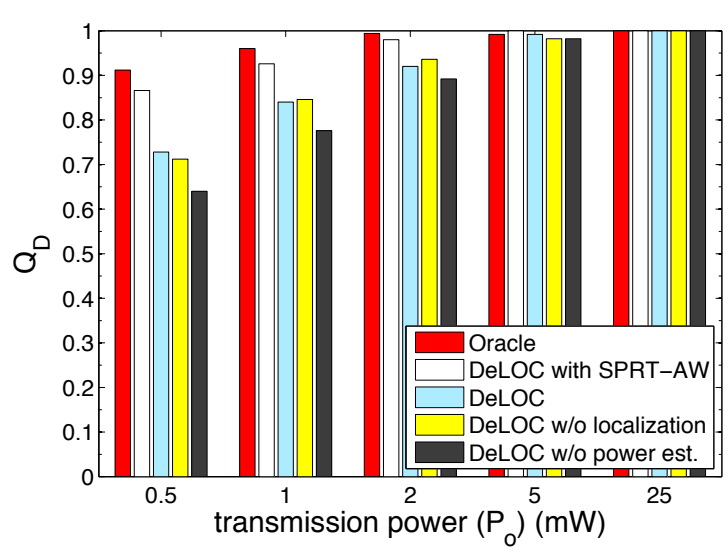

(b) Achieved detection probability

Fig. 7. Performance of DeLOC: DeLOC (a) requires only a small number of sensing rounds for WM detection, and (b) achieves a high detection rate even for a very weak signal power, e.g., $P_{o}=1 \mathrm{~mW}$.

These simulation results clearly demonstrate that the joint design of data-fusion and location \& transmit-power estimation maximizes the benefits of spatial-temporal sensing for detecting small-scale primaries, such as WMs in 802.22.

\section{CONCLUSION}

The detection of small-scale primary signals is a critical, but challenging problem in realizing opportunistic spectrum access in CRNs. To address this problem, we proposed a novel spatio-temporal fusion scheme that exploits (i) spatial diversity by cooperative sensing with an optimal fusion range, and (ii) temporal diversity by scheduling a series of sensing stages with an optimal stopping time. We modeled the detection problem as a hypothesis test, approximated the sum of sensor readings as a log-normal random variable, and then solved a convex optimization problem, to obtain the optimal fusion range that minimizes the average detection delay. We also proposed a new sensing algorithm called DeLOC that iterates between cooperative sensing and location/transmit-power estimation to further improve the sensing performance under realistic settings. Our evaluation results show that DeLOC reduces the detection delay significantly while achieving high detection performance.

\section{APPENDIX}

\section{A. Proof of Proposition 1}

To minimize the average number of sensing rounds $N$ in Eq. (8), we need to find the data-fusion range that maximizes the expected test statistic, i.e., $\mathbb{E}\left[\lambda \mid \mathcal{H}_{k}\right]$. Note that $\mathbb{E}\left[\Lambda_{N}\right]$ is a function of desired false-alarm and mis-detection probability, and does not depend on the fusion range, as indicated in Eq. (10). Let $\mathcal{J}\left(R_{f}\right) \triangleq \mathbb{E}\left[\lambda \mid \mathcal{H}_{1}\right]$ in Eq. (19). Then, we have:

$$
\begin{aligned}
\mathcal{J}\left(R_{f}\right) & \triangleq \mathbb{E}\left[\lambda \mid \mathcal{H}_{1}\right] \\
& =\frac{1}{2}\left(\phi_{0}^{2}-\phi_{1}^{2}\right) \rho \pi R_{f}^{2}+\frac{\phi_{1}-\phi_{0}}{\sigma_{n}}\left(P_{o} e^{\frac{1}{2} \sigma^{2}} m_{1}+N_{o} \rho \pi R_{f}^{2}\right) \\
& =a_{1} R_{f}^{2-\alpha}+a_{2} R_{f}^{2}+a_{3},
\end{aligned}
$$

where

$$
\begin{aligned}
& a_{1}=\frac{2\left(\phi_{1}-\phi_{0}\right) P_{o} e^{\frac{1}{2} \sigma^{2}} \rho \pi d_{o}^{\alpha}}{\sigma_{n}(2-\alpha)}, \\
& a_{2}=\frac{1}{2}\left(\phi_{0}^{2}-\phi_{1}^{2}\right) \rho \pi+\frac{\left(\phi_{1}-\phi_{0}\right) N_{o} \rho \pi}{\sigma_{n}}, \\
& a_{3}=\frac{2\left(\phi_{1}-\phi_{o}\right) P_{o} e^{\frac{1}{2} \sigma^{2}} \rho \pi d_{o}^{\alpha}}{\sigma_{n}(\alpha-2) \epsilon^{\alpha-2}} .
\end{aligned}
$$

To find an optimal value of $R_{f}$, we need to show the concavity of $\mathcal{J}\left(R_{f}\right)$ w.r.t. $R_{f}$. The first order derivative of $\mathcal{J}\left(R_{f}\right)$ is given as: 


$$
\frac{\partial \mathcal{J}\left(R_{f}\right)}{\partial R_{f}}=a_{1}(2-\alpha) R_{f}^{1-\alpha}+2 a_{2} R_{f} .
$$

Then, the second order derivative is given as:

$$
\begin{aligned}
& \frac{\partial^{2} \mathcal{J}\left(R_{f}\right)}{\partial R_{f}^{2}}=a_{1}(2-\alpha)(1-\alpha) R_{f}^{-\alpha}+2 a_{2} \\
& =\frac{4\left(\phi_{1}-\phi_{0}\right) \rho \pi}{\sigma_{n}}\left((1-\alpha) P_{o} e^{\frac{1}{2} \sigma^{2}}\left(\frac{d_{o}}{R_{f}}\right)^{\alpha}+N_{o}-\frac{\left(\phi_{1}+\phi_{0}\right) \sigma_{n}}{2}\right) \\
& \approx 2 \rho \pi(1-2 \alpha)\left(\frac{P_{R}}{\sigma_{n}}\right)^{2} .
\end{aligned}
$$

In practice, $\alpha>\frac{1}{2}$, so it is easy to show that $\frac{\partial^{2} \mathcal{J}\left(R_{f}\right)}{\partial R_{f}^{2}}<0$ and hence $\mathcal{J}\left(R_{f}\right)$ is concave. Therefore, the optimal fusion range $R_{f}$ can be derived from Eq. (24) as:

$$
R_{f}^{*}=\arg \max _{R_{f}} \mathcal{J}\left(R_{f}\right)=\left.R_{f}\right|_{\frac{\partial \mathcal{J}\left(R_{f}\right)}{\partial R_{f}}=0}=\left(\frac{a_{1}(\alpha-2)}{2 a_{2}}\right)^{\frac{1}{\alpha}} .
$$

Thus, the proposition holds.

\section{REFERENCES}

[1] FCC, "Second report and order," FCC 08-260, Nov 2008.

[2] IEEE 802.22 Working Group on Wireless Regional Area Networks, http://www.ieee802.org/22/.

[3] A. Ghasemi and E. S. Sousa, "Collaborative spectrum sensing for opportunistic access in fading environments," in Proc. IEEE DySPAN, Nov 2005.

[4] Y.-C. Liang, Y. Zeng, E. C. Y. Peh, and A. T. Hoang, "Sensingthroughput tradeoff for cognitive radio networks," IEEE Trans. Wireless Commun., vol. 7, pp. 1326-1337, April 2008.

[5] A. W. Min and K. G. Shin, "Impact of mobility on spectrum sensing in cognitive radio networks," in Proc. ACM CoRoNet, Sep 2009.

[6] A. W. Min, K. G. Shin, and X. Hu, "Attack-tolerant distributed sensing for dynamic spectrum access networks," in Proc. IEEE ICNP, Oct 2009.

[7] S. M. Mishra, R. Tandra, and A. Sahai, "Coexistence with primary users of different scales," in Proc. IEEE DySPAN, April 2007.

[8] D. Gurney, G. Buchwald, L. Ecklund, S. Kuffner, and J. Grosspietsch, "Geo-location database techniques for incumbent protection in the TV white space," in Proc. IEEE DySPAN, Oct 2008.

[9] FCC, "Notice of proposed rulemaking and order," FCC 08-188, Aug 2008.

[10] E. Reihl, "Wireless microphone characteristics," IEEE 802.2206/0070r0, May 2006.

[11] P. Bahl, R. Chandra, T. Moscibroda, R. Murty, and M. Welsh, "White space networking with Wi-Fi like connectivity," in Proc. ACM SIGCOMM, Aug 2009.

[12] OET Report FCC/OET 08-TR-1005, "Evaluation of the performance of prototype TV-band white space devices phase II," Technical Research Branch Laboratory Division, Office of Engineering and Technology, FCC, Oct 2008.

[13] G. Chouinard, "WRAN reference model," IEEE 802.22-04/0002r12, Sep 2005.

[14] — , "Sensing performance with the 802.22.1 wireless microphone beacon," IEEE 802.22-09/0068r1, Mar 2009.

[15] Z. Lei and F. Chin, "A reliable and power efficient beacon structure for cognitive radio systems," in Proc. IEEE ICC, May 2008.

[16] A. Ghasemi and E. S. Sousa, "Interference aggregation in spectrumsensing cognitive wireless networks," IEEE J. Sel. Topics Signal Process., vol. 2, no. 1, pp. 41-56, Feb 2008.

[17] G. Chouinard, "Wireless microphone sensing," IEEE 802.22-07/0530r1, Nov 2007.

[18] Kim et al., "Fractional BW usage for WRAN systems," IEEE 802.2206/0117r0, July 2006.

[19] Y. Zeng and Y.-C. Liang, "Simulations for wireless microphone detection by eigenvalue and covariance based methods," IEEE 802.22 07/0325r0, July 2007.

[20] H.-S. Chen, W. Gao, and D. G. Daut, "Spectrum sensing for wireless microphone signals," in Proc. IEEE SECON, June 2008.

[21] W. Rose, "Enhanced protection for low power licensed devices operating in tv broadcast bands," IEEE 802.22-06/0073r2, May 2006.

[22] L. Lai, Y. Fan, and H. V. Poor, "Quickest detection in cognitive radio: A sequential change detection framework," in Proc. IEEE Globecom, Nov 2008.
[23] A. W. Min and K. G. Shin, "An optimal sensing framework based on spatial RSS-profile in cognitive radio networks," in Proc. IEEE SECON, June 2009.

[24] R. Chen, J.-M. Park, and K. Bian, "Robust distributed spectrum sensing in cognitive radio networks," in Proc. IEEE INFOCOM, April 2008, pp. $1876-1884$

[25] R. Chen, J.-M. Park, and J. H. Reed, "Defense against primary user emulation attacks in cognitive radio networks," IEEE J. Sel. Areas Commun., vol. 26, no. 1, pp. 25-37, Jan 2008.

[26] G. Xing, R. Tan, B. Liu, J. Wang, X. Jia, and C.-W. Yi, "Data fusion improves the coverage of wireless sensor networks," in Proc. ACM MobiCom, 2009.

[27] C. R. Stevenson, C. Cordeiro, E. Sofer, and G. Chouinard, "RAN requirements," IEEE 802.22-05/0007r46, Sep 2005.

[28] S. Shellhammer, S. Shankar, R. Tandra, and J. Tomcik, "Performance of power detector sensors of DTV signals in IEEE 802.22 WRANs," in Proc. ACM TAPAS, Aug 2006.

[29] S. Shellhammer et al., "Spectrum sensing simulation model," IEEE 802.22-06/0028r10, Sep 2006.

[30] A. Taherpour, Y. Norouzi, M. Masiri-Kenari, A. Jamshidi, and Z. Zeinalpour-Yazdi, "Asymptotically optimum detection of primary user in cognitive radio networks," IET Communications, vol. 1, no. 6, pp. 1138-1145, Dec 2007.

[31] A. Wald, Sequential Analysis. Dover Publications, 2004.

[32] R. Menon, R. M. Buehrer, and J. H. Reed, "On the impact of dynamic spectrum sharing techniques on legacy radio systems," IEEE Trans. Wireless Commun., vol. 7, no. 11, pp. 4198-4207, Nov 2008.

[33] X. Hong, C.-X. Wang, and J. Thompson, "Interference modeling of cognitive radio networks," in Proc. IEEE VTC-spring, May 2008.

[34] S. M. Mishra, A. Sahai, and R. W. Brodersen, "Cooperative sensing among cognitive radios," in Proc. IEEE ICC, June 2006.

[35] L. Xiao, L. J. Greenstein, and N. B. Mandayam, "Sensor-assisted localization in cellular systems," IEEE Trans. Wireless Commun., vol. 6 , no. 12, pp. 4244-4248, Dec 2007.

[36] S. Liu, Y. Chen, W. Trappe, and L. J. Greenstein, "Non-interactive localization of cognitive radios based on dynamic signal strength mapping," in Proc. IEEE/IFIP WONS, Feb 2009.

[37] Z. Ma, W. C. K. B. Letaief, and Z. Cao, "A semi range-based iterative localization algorithm for cognitive radio networks," IEEE Trans. Veh. Technol., vol. 59, pp. 704-717, Feb 2010.

[38] M. Zafer, B. Ko, and I. W.-H. Ho, "Cooperative transmit-power estimation under wireless fading," in Proc. ACM MobiHoc, May 2008.

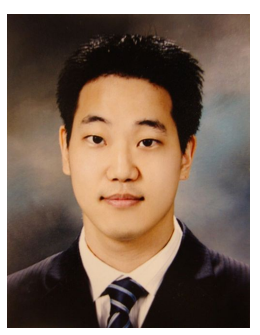

Alexander W. Min (S'08) received his B.S. degree in electrical engineering from Seoul National University, Korea, in 2005 and the M.S. degree from the University of Michigan in 2007. He is currently a Ph.D. candidate in the Department of Electrical Engineering and Computer Science, The University of Michigan, Ann Arbor. In 2010, he was a Research Intern at Deutsche Telekom Inc., R\&D Laboratories, Los Altos, USA. His research interests are in the area of cognitive radio and dynamic spectrum access networks including spectrum sensing, resource allocation, security, and secondary spectrum market. He is a student member of ACM and the IEEE Communications Society.

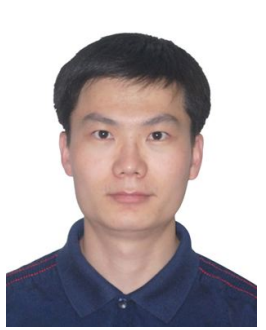

Xinyu Zhang received his B.Eng. degree in 2005 from Harbin Institute of Technology, China, and his M.S. degree in 2007 from the University of Toronto, Canada. He is currently a Ph.D. student in the Department of Electrical Engineering and Computer Science, University of Michigan. His research interests are in the design, optimization, and performance analysis of wireless network protocols, particularly with applications in software radio and cognitive radio networks. 


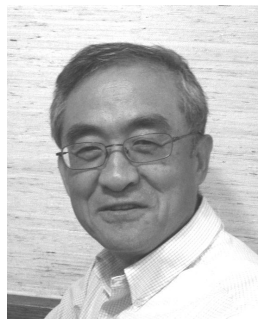

Kang G. Shin (F'92) is the Kevin and Nancy O'Connor Professor of Computer Science and Founding Director of the Real-Time Computing Laboratory in the Department of Electrical Engineering and Computer Science, The University of Michigan, Ann Arbor, Michigan. His current research focuses on QoS-sensitive networking and computing as well as on embedded real-time OS, middleware and applications, all with emphasis on timeliness, security, and dependability. He has supervised the completion of $66 \mathrm{PhDs}$, and authored/coauthored more than 740 technical articles. He has co-authored (jointly with C. M. Krishna) a textbook "Real-Time Systems," McGraw Hill, 1997.

$\mathrm{He}$ has received a number of best paper awards, including the IEEE Communications Society William R. Bennett Prize Paper Award in 2003, the Best Paper Award from the IWQoS'03 in 2003, and an Outstanding IEEE Transactions of Automatic Control Paper Award in 1987. He has also coauthored papers with his students which received the Best Student Paper Awards from the 1996 IEEE Real-Time Technology and Application Symposium, and the 2000 and 2010 UNSENIX Technical Conferences. $\mathrm{He}$ has also received several institutional awards, including the Research Excellence Award in 1989, Outstanding Achievement Award in 1999, Service Excellence Award in 2000, Distinguished Faculty Achievement Award in 2001, and Stephen Attwood Award in 2004 from The University of Michigan; a Distinguished Alumni Award of the College of Engineering, Seoul National University in 2002; 2003 IEEE RTC Technical Achievement Award; and 2006 Ho-Am Prize in Engineering.

He received the B.S. degree in Electronics Engineering from Seoul National University, Seoul, Korea in 1970, and both the M.S. and Ph.D degrees in Electrical Engineering from Cornell University, Ithaca, New York in 1976 and
1978, respectively. From 1978 to 1982 he was on the faculty of Rensselaer Polytechnic Institute, Troy, New York. He has held visiting positions at the U.S. Airforce Flight Dynamics Laboratory, AT\&T Bell Laboratories, Computer Science Division within the Department of Electrical Engineering and Computer Science at UC Berkeley, and International Computer Science Institute, Berkeley, CA, IBM T.J. Watson Research Center, Software Engineering Institute at Carnegie Mellon University, and HP Research Laboratories. $\mathrm{He}$ also chaired the Computer Science and Engineering Division, EECS Department, The University of Michigan for three years beginning January 1991.

$\mathrm{He}$ is Fellow of IEEE and ACM, and member of the Korean Academy of Engineering, is serving as the General Co-Chair for 2009 ACM Annual International Conference on Mobile Computing and Networking (MobiCom'09), was the General Chair for 2008 IEEE Communications Society Conference on Sensor, Mesh and Ad Hoc Communications and Networks (SECON'08), the 3rd ACM/USENIX International Conference on Mobile Systems, Applications, and Services (MobiSys'05) and 2000 IEEE Real-Time Technology and Applications Symposium (RTAS'00), the Program Chair of the 1986 IEEE Real-Time Systems Symposium (RTSS), the General Chair of the 1987 RTSS, the Guest Editor of the 1987 August special issue of IEEE Transactions on Computers on Real-Time Systems, a Program Co-Chair for the 1992 International Conference on Parallel Processing, and served numerous technical program committees. He also chaired the IEEE Technical Committee on Real-Time Systems during 1991-93, was a Distinguished Visitor of the Computer Society of the IEEE, an Editor of IEEE Trans. on Parallel and Distributed Computing, and an Area Editor of International Journal of Time-Critical Computing Systems, Computer Networks, and ACM Transactions on Embedded Systems. 